\title{
ESR STUDY OF THE MAGNETIC PROPERTIES OF THE CdTe/CdMnTe MULTI QUANTUM WELLS
}

\author{
M. SURma, M. Godlewski \\ Institute of Physics, Polish Academy of Sciences \\ Al. Lotników 32/46, 02-668 Warszawa, Poland \\ AND A. WAAG \\ Physikalisches Institut der Universität Würzburg \\ Am Ifubland, 8700 Würzburg, Germany
}

\begin{abstract}
The results of electron spin resonance investigations of bulk $\mathrm{Cd}_{1-x} \mathrm{Mn}_{x} \mathrm{Te}$ and of molecular beam epitaxy grown CdTe/CdMnTe single $2 \mu \mathrm{m}$ thick layer and multi quantum well with $\mathrm{Mn}$ concentrations of about $x=0.10$ are compared. The $\mathrm{Mn}^{2+}$ electron spin resonance spectrum of the MQW $\mathrm{CdTe} / \mathrm{CdMnTe}$ shows severalfeatures different from those observed in the CdMnTe bulk sample. The $\mathrm{Mn}^{2+}$ resonance shows a small anisotropy of position and widthwith the anisotropy axis normal to the heterointerface. The temperature dependence of the width of the electron spin resonance line is also different from that observed for the bulk and for the thick single layer.
\end{abstract}

PACS numbers: 73.20.Dx, 75.70.Fr, 76.30.Fc

\section{Introduction}

The purpose of this work was to compare magnetic properties ofbulk and multi quantum well (MQW) ternary CdMnTe compound with Mn fraction of $9.5 \%$ for the bulk sample and $10 \%$ for the two-dimensional (2D) system. The molecular beam epitaxy grown CdTe/CdMnTe MQW system (CT411) was grown on (100) CdTe with $6000 \AA$ thick CdMnTe buffer and consisted of $6 \mathrm{CdTe}$ quantum wells of different thicknesses $(6,12,18,28,60$ and $100 \AA)$ separated by $500 \AA \mathrm{Cd}_{1-x} \mathrm{Mn}_{x} \mathrm{Te}$ barriers and was covered with $1000 \AA$ thick CdMnTe upper layer. The CT608a sample consisted of a single $2 \mu \mathrm{m} \mathrm{CdMnTe} \mathrm{layer} \mathrm{grown} \mathrm{on} \mathrm{(100)} \mathrm{CdTe} \mathrm{substrate}$ separated by $2000 \AA$ CdTe buffer. 


\section{ESR experiments}

The $\mathrm{Mn}^{2+}$ electron spin resonance (ESR) spectrum of CT411 and CT608a samples was relatively weak and broad (22 $\mathrm{mT}$ (CT411) and $20 \mathrm{mT}$ (CT608a) at $100 \mathrm{~K}$ ). For the CT411 sample the ESR signal was observed for low temperature below $110 \mathrm{~K}$. The ESR signal of the CT411 sample was at $100 \mathrm{~K}$ about $5 \mathrm{mT}$ broader than that for the $9.5 \% \mathrm{CdMnTe}$ bulk sample. This indicates some inhomogeneous broadening of the Mn resonance in the MQW system, which we relate tentatively to $\mathrm{Mn}$ diffusion at the $\mathrm{CdTe} / \mathrm{CdMnTe}$ interfaces. We have also observed a $5 \%$ anisotropy of the ESR signal width. Figure 1 shows the temperature dependence of the ESR spectra of the bulk and of the two MBE samples. The

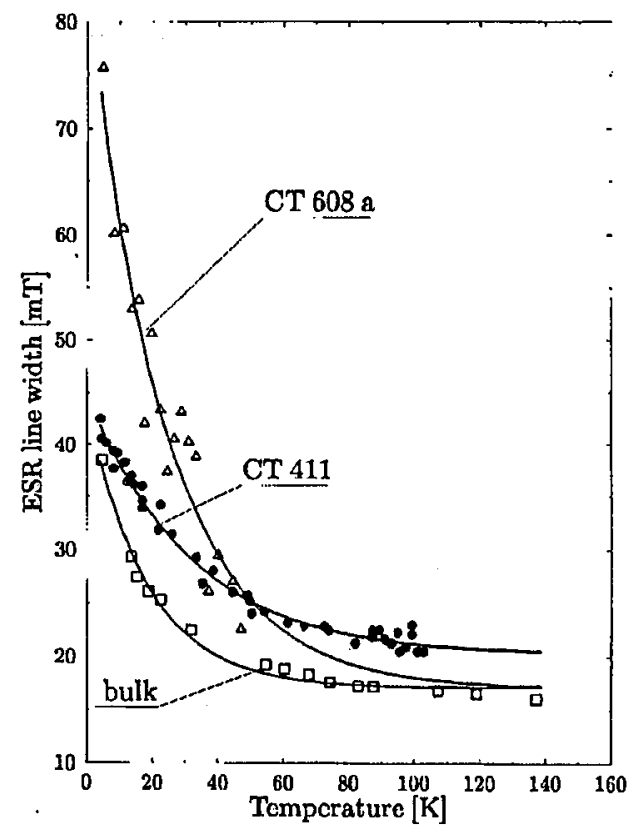

Fig. 1. Temperature dependence of $\mathrm{Mn}^{2+}$ ESR signal of the two MBE-grown samples: MQW system (CT411), single thick layer (CT608a), and of the bulk CdMnTe. Solid lines present a fit to the experimental data with semiempirical formulae described in the text.

$\Delta H_{\mathrm{pp}}(T)$ dependence of the MQW sample is different from those observed for the bulk and the single layer sample. For the two latter samples the signal width was the same at room temperature but the dependence spreads at low temperatures, due to larger line width of the $\mathrm{Mn}$ resonance in the CT608a MBE layer. The temperature dependence for an MQW sample is weaker, which probably is also due to the Mn diffusion at the $\mathrm{CdTe} / \mathrm{CdMnTe}$ interface.

The $\mathrm{Mn}^{2+}$ resonance in the bulk CdMnTe sample is isotropic. This is not the case of the MBE samples, as shown in Fig. 2. The anisotropy observed is a 


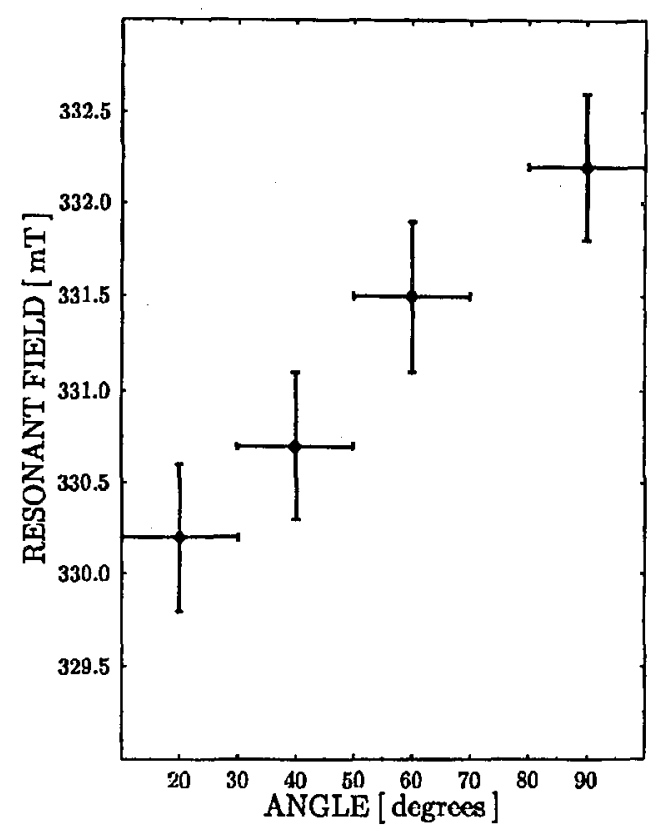

Fig. 2. Anisotropy of the $\mathrm{Mn}^{2+} \mathrm{ESR}$ signal in the MQW system. The spectra were measured at $12.4 \mathrm{~K}$ for the sample rotated between two extreme configurations of the magnetic field versus the interface axis. For 90 degrees the magnetic field was normal to the interface plane.

characteristic feature of heterojunction systems with the lattice constant misfit [1]. This means that even though a thick $6000 \AA \mathrm{CdMnTe}$ buffer was used, some residual strain is still left in the MQW structure. The observed signal anisotropy could also come from the $\mathrm{Mn}^{2+}$ ions at the well-barrier interfaces. However, in the ESR study of the single CdMnTe layer a similar signal anisotropy was observed, which allows us to reject the alternative explanation.

A residual strain in the structures has also been determined by X-ray diffraction measurements. For that reciprocal space mapping of asymmetric reflections has been performed. A strain parameter $\Gamma$ has been deduced. $\Gamma=1$ corresponds to a fully strained layer, whereas $\Gamma=0$ describes a fully relaxed layer. The strain parameter $\Gamma$ is given by

$$
\frac{(a(\text { layer })-a(\text { sub }))_{\text {parallel }}}{a(\text { sub })}=(1-\Gamma) \frac{(a(\text { layer })-a(\text { sub }))_{\text {relaxed }}}{a(\text { sub })},
$$

where $a($ layer $), a($ sub $)$ are lattice constants of epilayer and substrate, respectively. A possible tilt of the substrate and layer orientation has been taken into account for the analysis. For the thick CdMnTe layer CT608, a strain parameter $\Gamma$ of 0.6 has been measured, which indeed means that the CdMnTe layer is still partly strained. 


\section{Temperature dependence of the line width}

Application of the ESR method to characterization of Mn based ternary compounds was reviewed by Oseroff and Keesom [2]. It was observed by several authors that the ESR signal line width $\left(\Delta H_{\mathrm{pp}}\right)$ increases with decreasing temperature. Three different approaches were proposed to describe signal broadening [3-5]. These were the Huber [3], Dormann and Jaccarino [4] and Bhagat [5] formulae. $T_{\mathrm{c}}$ parameter in each of these formulae was related to the order-disorder critical $[3,4]$ and spin freezing [5] temperatures, i.e., ESR can be used to estimate magnetic phase transition temperature.

Several authors argued an advantage of a particular description of the ESR data and physical meaning of the parameters [6-8]. Ilowever, no consensus was reached which of the approaches is "the correct one" [2]. It is why we used these three formulae to fit the experimental data. The IIuber formula gave $T_{\mathrm{c}} \approx 0 \mathrm{~K}$ for the bulk sample, i.e., the critical temperature agrees with the magnetic phase transition temperature. This is in contrary to some earlier statements that the Huber equation describes the data only far above the critical temperature [2]. The similar value of the critical temperature was also obtained from the Dormann and Jaccarino formula. However, the Curie-Weiss temperature of $0.15 \mathrm{~K}$ obtained from the fit is too small [6]. Formally the best fit to the data (the smallest standard deviation) was obtained with the empirical Bhagat formula but with the $18.2 \mathrm{~K}$ critical temperature. Even though the temperature dependence of the ESR line width of the MQW system looked differently, the fit with two first formulae gave $T_{c}$ values about $0 \mathrm{~K}$, i.e., the same as for the bulk sample.

\section{Acknowledgments}

We want to thank H. Heinke for the X-ray diffraction measurements.

\section{References}

[1] E. Glaser, T.A. Kennedy, R. Sillmon, M.G. Spencer, Phys. Rev. B 40, 3447 (1989).

[2] S. Oseroff, P.H. Keesom, in: Semiconductors and Semimetals, Eds. R.K. Willardson, A.C. Beer, Vol. 25, Academic Press, New York 1988, p. 73.

[3] D.L. Huber, Phys. Rev. B 6, 3180 (1972).

[4] E. Dormann, V. Jaccarino, Phys. Lett. A 48, 81 (1974).

[5] S.M. Bhagat, M.L. Spano, J.N. Lloyd, Solid State Commun. 38, 261 (1981).

[6] S. Oseroff, M. Mesa, M. Tovar, R. Arce, J. Appl. Phys. 53, 2208 (1982).

[7] R.E. Kremer, J.K. Furdyna, J. Magn. Magn. Mater. 40, 185 (1983).

[8] D.J. Webb, S.M. Bhagat, J.K. Furdyna, J. Appl. Phys. 55, 2310 (1984). 